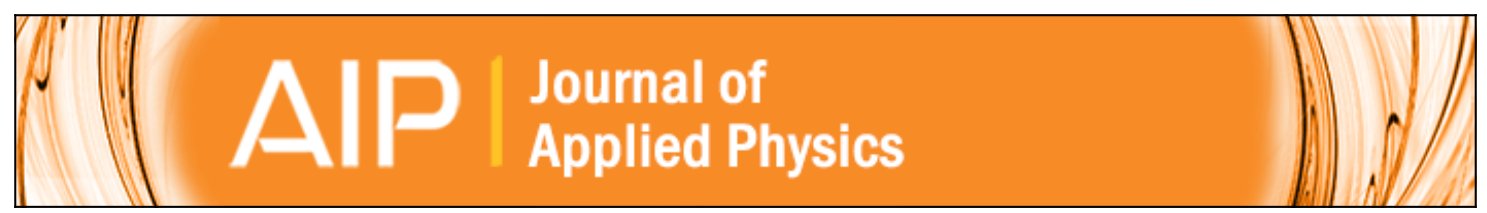

\title{
Tunneling Currents in Zinc Oxide
}

R. C. Neville and C. A. Mead

Citation: Journal of Applied Physics 41, 5285 (1970); doi: 10.1063/1.1658664

View online: http://dx.doi.org/10.1063/1.1658664

View Table of Contents: http://scitation.aip.org/content/aip/journal/jap/41/13?ver=pdfcov

Published by the AIP Publishing

\section{Articles you may be interested in}

Dissimilar-electrodes-induced asymmetric characteristic and diode effect of current transport in zinc oxide tunnel junctions

J. Appl. Phys. 114, 044111 (2013); 10.1063/1.4816796

Current-driven hydrogen incorporation in zinc oxide

Appl. Phys. Lett. 91, 212102 (2007); 10.1063/1.2816119

Magnetic tunnel junctions with a zinc oxide-cobalt oxide composite tunnel barrier

Appl. Phys. Lett. 86, 112505 (2005); 10.1063/1.1882762

Polarization currents in zinc oxide varistors from 77 to $450 \mathrm{~K}$

J. Appl. Phys. 76, 7367 (1994); 10.1063/1.357960

Prebreakdown conduction in zinc oxide varistors: Thermionic or tunnel currents and onestep or twostep conduction processes

Appl. Phys. Lett. 63, 1077 (1993); 10.1063/1.109838

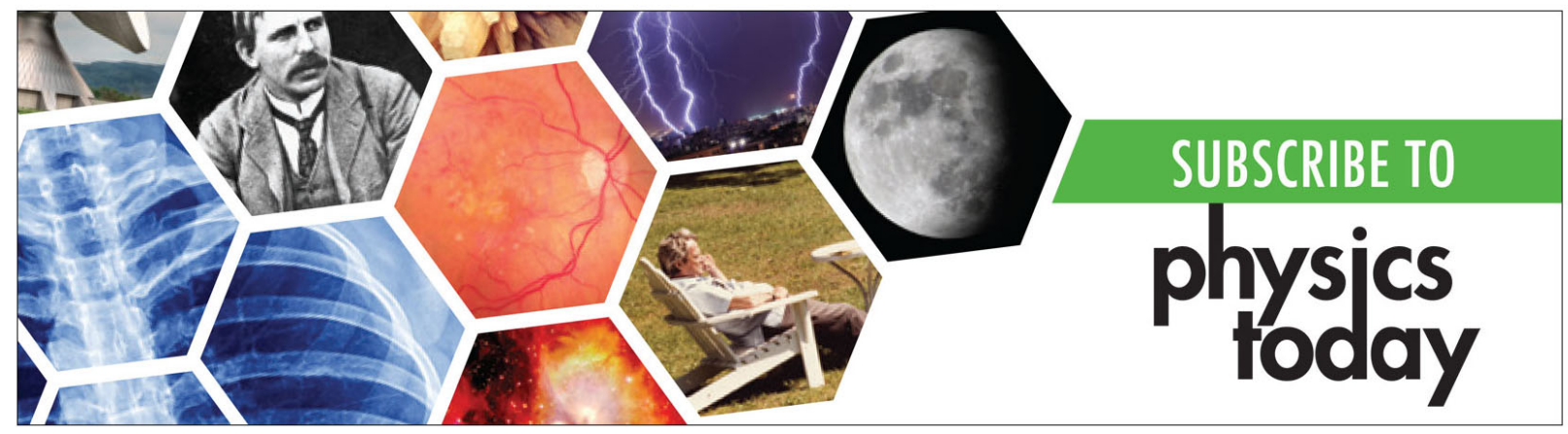


${ }^{9}$ E. Schibli and A. G. Milnes, Mater. Sci. Eng. 2, 173 (1967).

${ }_{10}$ B. I. Boltaks, Difusion in Semiconductors (Academic, New York, 1963), Chap. 7.

11 L. J. van der Pauw, Phillips Res. Repts. 13, 1 (1958).

12 E. M. Conwell, Proc. IRE 46, 1281 (1958).
${ }^{13}$ Althou, $h$ this lack of $0.53-\mathrm{eV}$ threshold in photoconductivity and absorption is not understood theoretically, it is important to the existence of negative photoconductivity. This point is discussed further by M. C. P. Chang, C. M. Penchina, and J. S. Moore (unpublished).

\title{
Tunneling Currents in Zinc Oxide
}

\author{
R. C. Neville and C. A. Mead \\ California Institute of Technology, Pasadena, California 91109 \\ (Received 15 June 1970; in final form 10 August 1970)
}

\begin{abstract}
An examination of the current-voltage characteristics of gold and palladium surface barriers on degenerate zinc oxide has been made. Both chemically prepared and cleaved surfaces were studied. The current conduction mode is shown to be thermionic-field emission at room temperature and to be pure field emission at liquid-nitrogen temperatures. The voltage dependence of the current is in good agreement with theory. The observed current magnitudes in both current modes were approximately one-tenth that calculated by simple theory.
\end{abstract}

\section{INTRODUCTION}

The characteristics of Schottky barriers on nondegenerate zinc oxide have been examined by $\mathrm{Mead}^{1}$ and Neville and $\mathrm{Mead}^{2}$ and have been found to be in good agreement with thermionic current theory. To gain additional understanding of the properties of zinc oxide as a semiconductor, studies of the properties of Schottky barriers on degenerate zinc oxide crystals were undertaken.

The theoretical aspects of current conduction for Schottky barriers on degenerate semiconductors have been treated by Padovani and Stratton, ${ }^{3}$ Conley and Mahan, ${ }^{4}$ Millea, McColl, and Mead, ${ }^{5}$ Parker and Mead ${ }^{6}$ and Parker ${ }^{7}$ among others. Expressions for both current magnitude and voltage dependence of the current have been developed. The above authors have considered not only pure field emission (tunneling) but have considered $^{3}$ the conditions under which a mixture of tunneling and thermionic processes take place. For sufficiently high temperatures current conduction is purely thermionic and occurs by means of electrons excited over the Schottky barrier. There is, however, a range of temperatures and carrier concentrations in which the temperature is sufficient to excite electrons to energies which are a significant fraction of the barrier energy. From this elevated energy the electrons tunnel through a barrier which is much reduced in thickness in comparison to the barrier at the conduction band edge. After Padovani and Stratton ${ }^{3}$ we call this mode thermionic-field emission.

Parker ${ }^{7}$ studied tunneling current modes in indium arsenide and cadmium telluride, effective masses $0.02 m_{e}$ and $0.11 m_{e}$, respectively, and found good agreement with theory. Millea et al..$^{5}$ studied tunneling in $n$-type GaAs $\left(m^{*} \approx 0.072 m_{e}\right)$ which was found to conform well with theory. In contrast to these materials, zinc oxide possesses a relatively heavy conduction band effective mass of $0.38 m_{e}{ }^{8} \mathrm{~A}$ study of zinc oxide afforded an examination of the theory as applied to relatively heavy-mass materials.

Indium-doped zinc oxide crystals were obtained with an average effective electron concentration between $2 \times 10^{19}$ and $4 \times 10^{19}$ per $\mathrm{cm}^{3}$ as determined by Hall and resistivity measurements. These values are well in excess of the effective conduction band density of states $\left(6 \times 10^{18}\right.$ per $\mathrm{cm}^{3}$ at $300^{\circ} \mathrm{K}$ and $8 \times 10^{17}$ per $\mathrm{cm}^{3}$ at $77^{\circ} \mathrm{K}$ ). Hall measurements at room and liquid-nitrogen temperatures were found to give values of mobility consistent with those of Rupprecht and Bogner,.$^{9,10}$ Carrier concentrations for individual barriers were found from capacitance at zero applied bias. These values were found to be consistent with concentration values determined from the Hall and resistivity measurements.

Forward current-voltage measurements were taken for some 40 individual surface barriers at both $300^{\circ}$ and $77^{\circ} \mathrm{K}$. The results were then examined in respect to simple tunneling theory., ${ }^{3,4,6,7}$ Both the current depend ence of voltage and the magnitudes of the currents were examined. Carrier concentration for individual barriers varied between $7 \times 10^{18}$ and $5.7 \times 10^{19}$ per $\mathrm{cm}^{3}$. Under these conditions the principle current mechanism is found to be thermionic-field emission at room temperature and to be pure field emission at liquid-nitrogen temperatures.

\section{SAMPLE PREPARATION}

Indium-doped hexagonal crystals of zinc oxide were cleaned by immersion in concentrated phosphoric acid for a period of $15 \mathrm{~min}$, followed by a 5 -min soak in concentrated hydrochloric acid, a rinse in running deionized water and then were dried with a jet of dry air.

For resistivity and Hall measurements the cleaned crystals were given five Ohmic contacts by soldering fine wires to the crystal with $90 \%$-indium and $10 \%$-silver solder.

After the resistivity and Hall measuremen were 


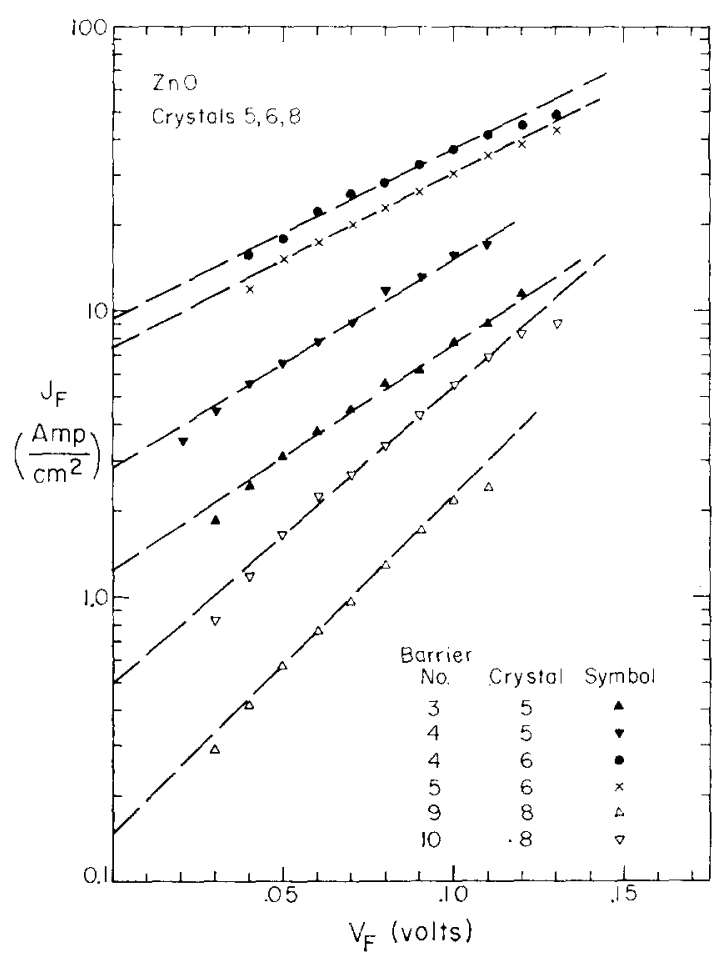

FIG. 1. Forward current density versus voltage at $300^{\circ} \mathrm{K}$ for barriers on zinc oxide of different carrier concentrations.

completed the indium solder was removed and the crystals recleaned in phosphoric acid, hydrochloric acid, and de-ionized water as above. The crystals were then placed in a vacuum chamber at a nominal pressure of $10^{-6}$ Torr. Approximately $1000 \AA$ of gold or of palladium were evaporated from a heated tungsten filament on to the zinc oxide crystals through a fine mesh screen. Nominal surface-barrier-contact diameter was $0.01 \mathrm{~cm}$. Contact to the evaporated barriers was made by a fine gold-wire probe in a three-dimensional micromanipulator. Bulk contacts were made with $90 \%$-indium and $10 \%$-silver solder. A three-point differential measurement was used to eliminate excessive voltage values due to the potential drop at the bulk crystal contacts.

After measurement of the barriers on the lateral surfaces, the zinc oxide crystals were cleaved in air along the basal plane. The crystals were immediately placed in a vacuum at a pressure of $\left\langle 10^{-6}\right.$ Torr. Barriers were evaporated, contacts made, and electrical measurements were made as for the chemically prepared surfaces.

\section{Initial Measurements}

Resistivity measurements were made on hexagonal indium-doped zinc oxide crystals at room temperature $\left(300^{\circ} \mathrm{K}\right)$ and at liquid-nitrogen temperature $\left(77^{\circ} \mathrm{K}\right)$. Hall measurements were made using a $4.7 \mathrm{kG}$ magnet at room temperature. The effective carrier concentration $w_{i}$ is determined from

$$
N_{d}=1 / q \mu \rho,
$$

where $N_{d}$ is the effective carrier density, $q$ is the electronic charge, $\mu$ is the mobility, assumed equal to the Hall mobility, and $\rho$ is the resistivity. Typical measured values of resistivity and mobility and the computed carrier concentration are presented in Table I.

The measured values of mobility were consistent with those reported by Rupprecht ${ }^{9}$ and Bogner. ${ }^{10}$ The carrier concentration obtained in this manner is clearly an average value. Departures of considerable magnitudecan be expected for localized regions, particularly in the neighborhood of the crystal surfaces.

Capacitance-voltage and voltage-current characteristics were taken for both gold and palladium barriers on both chemically prepared and freshly cleaved surfaces. In Fig. 1 we present plots of forward current density as a function of the forward voltage at $300^{\circ} \mathrm{K}$ for typical sample barriers on crystal samples 5, 6, and 8 . In Fig. 2 we present the same type of information at liquid-nitrogen temperatures. Pertinent data including capacitance values for these surface barriers is presented in Appendix A.

From the carrier concentration determined by resistivity and Hall effect it is seen that the zinc oxide crystals under investigation are sufficiently heavily doped to be degenerate.

Using a value of $0.38 m_{e}$ for the conduction band effective mass from Dietz et al..$^{8}$ the effective conduction band density of states is calculated to be $6 \times 10^{18}$ per $\mathrm{cm}^{3}$ at room temperature and $8 \times 10^{17}$ per $\mathrm{cm}^{3}$ at liquidnitrogen temperatures. The Fermi level lies between

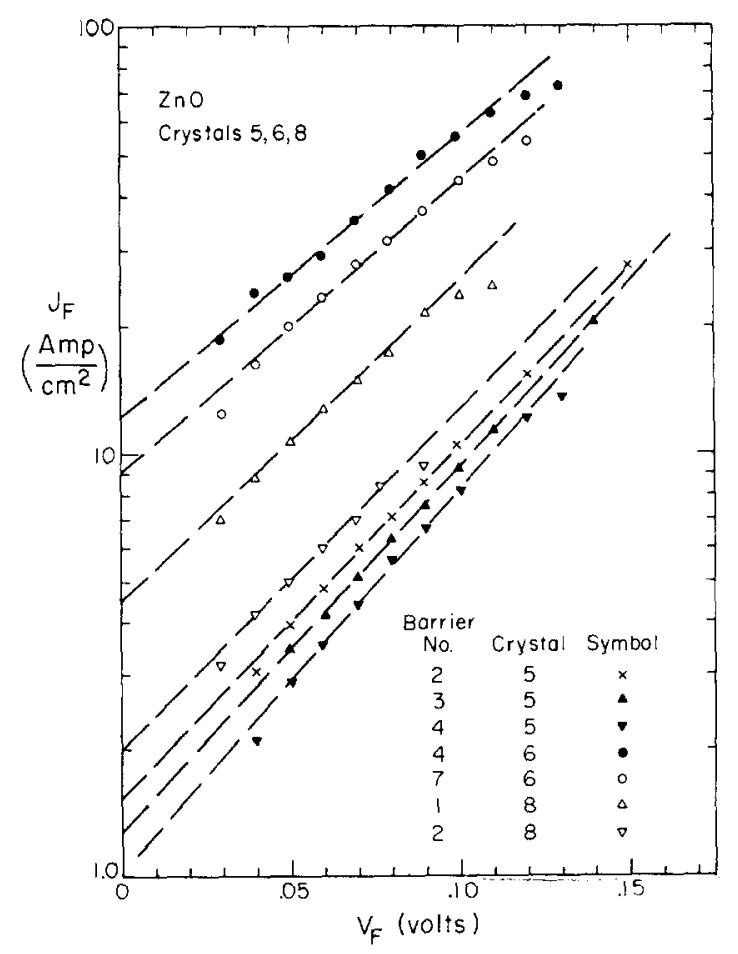

FIg. 2. Forward current density versus voltage at $77^{\circ} \mathrm{K}$ for barriers on zinc oxide crystals of different carrier concentrations. 
0.030 and $0.120 \mathrm{eV}$ above the conduction band edge at room temperature for carrier concentrations between $1 \times 10^{19}$ and $5 \times 10^{19}$ per $\mathrm{cm}^{3}$. At liquid-nitrogen temperature the degeneracy lies between 0.047 and 0.143 $\mathrm{eV}$ for the same range of carrier concentrations.

The carrier concentration of these indium-doped zinc oxide samples is sufficiently high to produce significant currents due to thermionic-field emission at room temperature and due to pure-field emission at liquidnitrogen temperatures. Current-voltage characteristics due to thermionic-field emission and to pure-field emission have been studied on other materials by Padovani and Stratton, ${ }^{3}$ Conley and Mahan, ${ }^{4}$ Millea, McColl, and Mead, ${ }^{5}$ and Parker. ${ }^{7}$

\section{Pure Tunneling Currents}

There are three separate modes to be considered for pure-field emission current depending on the energy distribution of the electrons available for the tunneling process. From Parker $^{7}$ we see that the case of interest here is that treated in detail by Millea, McColl, and Mead. ${ }^{5}$ In the case of interest here, the degeneracy $\zeta$ is greater than the change in energy $1 / S_{m}$ necessary to reduce the tunneling probability by $1 / e$. Both $\zeta$ and $1 / S_{m}$ are greater than the thermionic energy $k T$. This condition is stated as

$$
k T<1 / S_{m}<\zeta
$$

where $T$ is the absolute temperature and $k$ is the Boltzmann constant. For forward bias greater than a few $k T$ the current density $J$ is given approximately by

$$
J=J_{0} \exp S_{m} E,
$$

where $J_{0}$ is a slowly varying function of applied voltage, temperature, impurity concentration, and effective mass, $E$ is the applied voltage in energy units,

$$
S_{m}=(2 / \hbar q)\left(\epsilon m^{*} / N_{d}\right)^{1 / 2} \quad \text { is the slope, }
$$

$m^{*}$ is the effective mass, $\hbar$ is Planck's constant divided by $2 \pi$, and $\epsilon$ is the permittivity of zinc oxide, taken as $8 \epsilon_{0}$ after Ref. 11.

The pre-exponential factor $J_{0}$ in Eq. (3) is strongly effected by the energy distribution of electrons in the

TABLE I. Resistivity, mobility, and carrier concentration of typical zinc oxide specimens.

\begin{tabular}{cccc}
\hline $\begin{array}{c}\text { Sample crystal } \\
\text { number }\end{array}$ & 5 & 6 & 8 \\
\hline $\begin{array}{c}\text { Resistivity at } 296^{\circ} \mathrm{K} \\
(\Omega \mathrm{cm})\end{array}$ & $5.5 \times 10^{-3}$ & $3.0 \times 10^{-3}$ & $4.0 \times 10^{-3}$ \\
$\begin{array}{c}\text { Resistivity at } 77^{\circ} \mathrm{K} \\
(\Omega \mathrm{cm})\end{array}$ & $5.8 \times 10^{-3}$ & $3.2 \times 10^{-3}$ & $4.3 \times 10^{-3}$ \\
$\begin{array}{c}\text { Hall mobility at } 296^{\circ} \mathrm{K} \\
\left(\mathrm{cm}^{2} / \mathrm{V} \mathrm{sec}\right)\end{array}$ & 49 & 58 & 55 \\
$\begin{array}{c}\text { Concentration at } 296^{\circ} \mathrm{K} \\
\left(\mathrm{cm}^{-3}\right)\end{array}$ & $2.3 \times 10^{19}$ & $3.5 \times 10^{19}$ & $2.85 \times 10^{19}$ \\
\hline
\end{tabular}

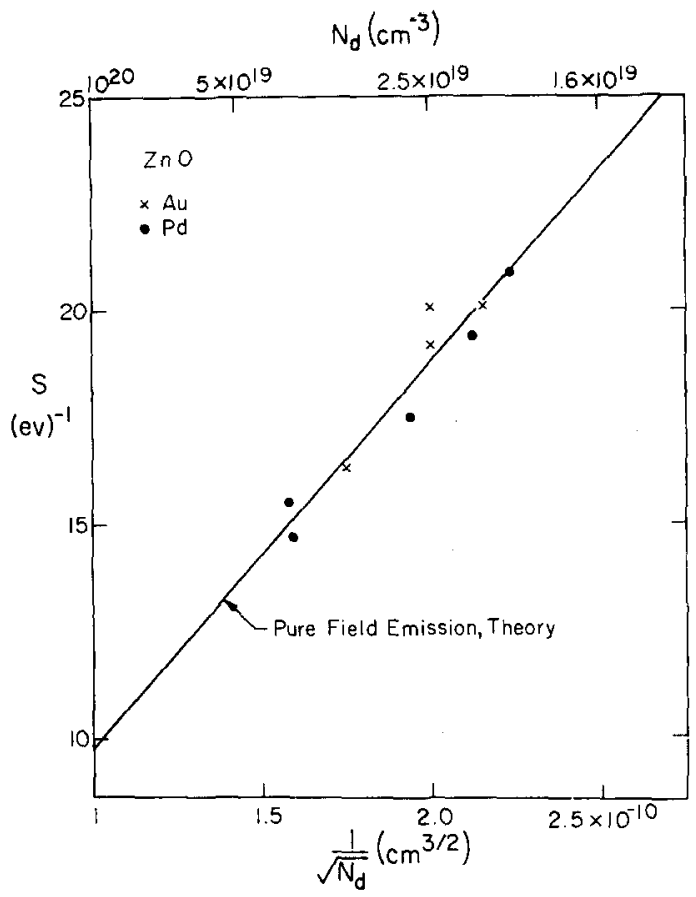

Frg. 3. Slope of forward $\log$ current versus voltage curve at $77^{\circ} \mathrm{K}$ as a function of carrier concentration. The theoretical value as calculated from Eq. (4) is given by the solid curve.

conduction band. For the electron supply function limited by $S_{m}$, it has been shown by Millea $e t a l .{ }^{5}$ that for a single-band model Eq. (3) becomes

$$
\begin{gathered}
J=J_{M} \exp \left(-S_{m} \Delta\right) \\
\Delta=\left(\phi_{B}-E\right)^{1 / 2}\left(\phi_{B}+0.6 \zeta-E\right)^{1 / 2} \\
-0.6 \zeta\left(\ln \frac{\left(\phi_{B}-E\right)^{1 / 2}+\left(\phi_{B}+0.6 \zeta-E\right)^{1 / 2}}{(0.6 \zeta)^{1 / 2}}\right),
\end{gathered}
$$

where $\phi_{B}$ is the barrier energy,

$$
\begin{gathered}
J_{M}=\left[A^{*} /\left(C_{1} k T\right)^{2}\right]\left[\pi C_{1} k T / \sin \pi C_{1} k T\right], \\
C_{1}=\left(S_{m} / 2\right) \ln \left[4\left(\phi_{B}-E\right) / 0.6 \zeta\right],
\end{gathered}
$$

and

$$
A^{*}=m^{*} q(k T)^{2} / 2 \pi^{2} \hbar^{3} \text { is the Richardson constant. }
$$

The upper temperature limit for pure field emission has been shown by Murphy and Good ${ }^{12}$ to be given by

$$
\left[C_{1}+\left(S_{m} / 2 \zeta\right)\right]^{-1}>k T,
$$

In Fig. 3 we present the measured slope $S_{m}$ plotted against the inverse square root of the donor concentration at liquid-nitrogen temperature for the barriers studied. Also presented is the theoretical slope $S_{m}$ from Eq. (4). Agreement is very good. The slopes found in Fig. 5 differ by more than a factor of 6 from the theoretical slope for thermionic operation, a value of $151 \mathrm{eV}^{-1}$ at $77^{\circ} \mathrm{K}$.

For pure field emission Parker and $\mathrm{Mead}^{6}$ have demonstrated that in the presence of trapping states the slope $S$ is one-half $S_{m}$ in the forbidden band. No evidence 


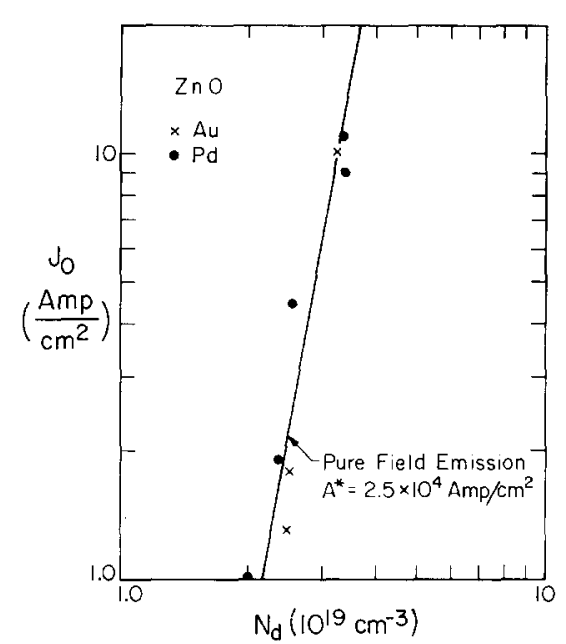

Fig. 4. Extrapolated zero bias current density versus carrier concentration at $77^{\circ} \mathrm{K}$. The solid curve is calculated based on Eqs. (5)-(7).

of such a phenomenon was found in our samples. When the log current versus voltage data given in Fig. 2 were extrapolated to higher current densities, the curves intersect at a voltage of approximately $0.65 \mathrm{~V}$; the approximate value of the barrier energy established previously by Neville and Mead. ${ }^{2}$ At $0.65 \mathrm{~V}$ the current density lies between 4 and $4.8 \times 10^{\mathrm{h}} \mathrm{A}$ per $\mathrm{cm}^{2}$.

In Fig. 4 we have plotted the logarithm of the extrapolated zero-bias current density at liquid-nitrogen temperatures versus the impurity concentration. Also

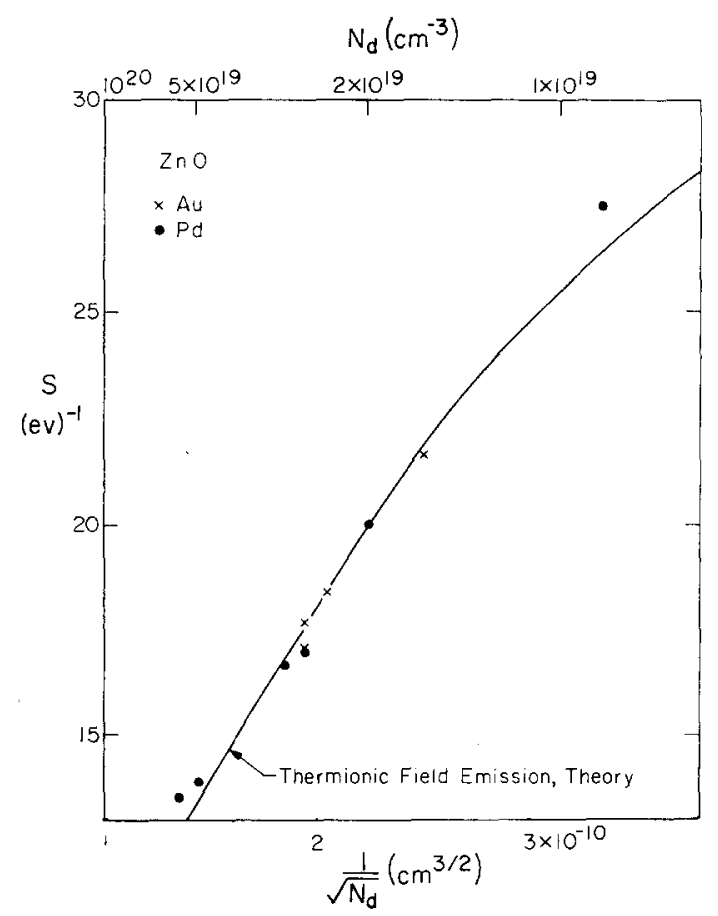

FIG. 5. Slope of forward log current versus voltage curve at $300^{\circ} \mathrm{K}$ as a function of carrier concentration. The theoretical value as calculated from $\mathrm{Eq}$. (14) is given by the solid curve. presented is the theoretical dependence using Eqs. (5) and (6). A value of $2.5 \times 10^{4} \mathrm{~A}$ per $\mathrm{cm}^{2}$ for the Richardson constant yields excellent agreement with the data. This value is approximately one-tenth that calculated for $A^{*}$ from simple theory, Eq. (9).

\section{Thermionic-Field Emission}

For thermionic-field emission Padovani and Stratton ${ }^{3}$ have shown that the current-voltage relationship is given by

$$
J=J_{0} \exp (S E)
$$

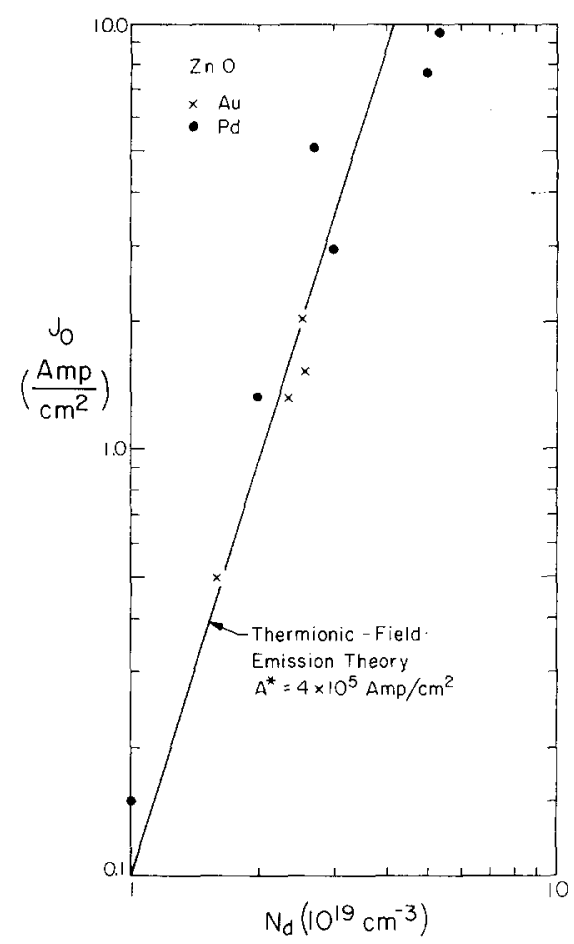

FIG. 6. Extrapolated zero bias current density versus carrier concentration at $300^{\circ} \mathrm{K}$. The solid curve is calculated based on Eqs. (11) $-(13)$.

where

$$
J_{0}=\left\{A^{*}\left(1 / S_{m} \pi\right)^{1 / 2}\left(\phi_{B}-V+\zeta\right)^{1 / 2} / k T \cosh \left[\left(1 / S_{m}\right) k T\right]\right\} \delta
$$

$$
\begin{gathered}
\delta=\exp \left\{\zeta /[k T]-S\left(\phi_{B}+\zeta\right)\right\} \\
S=S_{m} \tanh \left(1 / S_{m} k T\right) .
\end{gathered}
$$

The thermionic-field emission expressions (11)-(14) have been shown valid for temperatures satisfying ${ }^{3}$

$$
k T>\left(2 / S_{m}\right)\left\{\ln \left[4\left(\phi_{B}-V\right) / \zeta\right]\right\}^{-1} .
$$

An upper temperature limit is imposed by the onset of significant current due to thermal emission over the barrier. This upper temperature limit is given by ${ }^{3}$

$$
\frac{\operatorname{coth}\left(1 / S_{m} k T\right)}{\sinh \left(1 / S_{m} k T\right)}<2\left(\phi_{B}+\zeta-E\right) \frac{S_{m}}{3} \text {. }
$$


Equations (10), (15), and (16) are concerned with the temperature range of validity for the thermionicfield and pure field emission models. Applying these equations to the carrier concentrations of interest $\left(7 \times 10^{18}\right.$ to $6 \times 10^{19}$ per $\left.\mathrm{cm}^{3}\right)$ it can be seen that, for the zero bias condition, pure-field emission is valid at $300^{\circ} \mathrm{K}$ for concentration levels exceeding $5 \times 10^{19} \mathrm{per}^{\mathrm{cm}^{3}}$. The thermionic-field emission mode of current is valid for carrier concentrations between $6 \times 10^{18}$ and $3 \times 10^{19}$ per $\mathrm{cm}^{3}$. At room temperature the range of concentrations between $3 \times 10^{19}$ and $5 \times 10^{19}$ per $\mathrm{cm}^{3}$ is covered neither by the pure-field emission nor by the thermionic-field emission theories. We shall consider both current mechanisms as limiting cases for those experimental samples whose carrier concentrations fall in this range.

In Fig. 5 we present the measured slope $S$ of the current density versus voltage characteristic as a function of the inverse impurity concentration for the samples at room temperature. Also presented in Fig. 5 is the theoretical slope for thermal field emission as calculated from Eqs. (4) and (14). The effective mass used was $0.38 m_{e}$ after Dietz et $a l .{ }^{8}$ The quan titative agreement between thermal-field-emission theory and experiment is excellent. The slope values are quite different than the value 38.4 per $\mathrm{eV}$ measured for pure thermionic emission at $300^{\circ} \mathrm{K} .{ }^{2}$ No evidence is seen for current conduction via trapping states in the forbidden band.

The forward current density at room temperature was extrapolated to zero forward bias. This extrapolated current density is displayed as a function of impurity in Fig. 6. Included in Fig. 6 is the theoretical current density as determined from Eq. (10) using a value of $4 \times 10^{5} \mathrm{~A}$ per $\mathrm{cm}^{2}$, a value consistent with that at $77^{\circ} \mathrm{K}$ but a factor of 10 less than that given by simple theory [Eq. (9)].

\section{SUMMARY}

The forward current-voltage characteristics of gold and palladium surface barriers on indium-doped hexagonal zinc oxide crystals have been measured. Both the chemically prepared lateral surfaces and the air cleaved basal plane of the crystals were used.

Hall measurements of mobility indicate a value between 40 and $60 \mathrm{~cm}^{2}$ per $V$ sec consistent with values reported in the literature. ${ }^{9,10}$ Resistivity measurements using a four-point technique yielded values between $3 \times 10^{-3}$ and $6 \times 10^{-3} \Omega \mathrm{cm}$ for the various sample crystals both at room and at liquid-nitrogen temperatures. The resistivity increase for any single crystal between room and liquid-nitrogen temperatures was less than $10 \%$. By combining the room-temperature Hall and resistivity measurements, an average value of the carrier concentration, assumed equal to the donor density in the crystal, was obtained. The values obtained varied from $2 \times 10^{19}$ to $4 \times 10^{19}$ per $\mathrm{cm}^{3}$ at room temperature.

Capacitance measurements were made on the

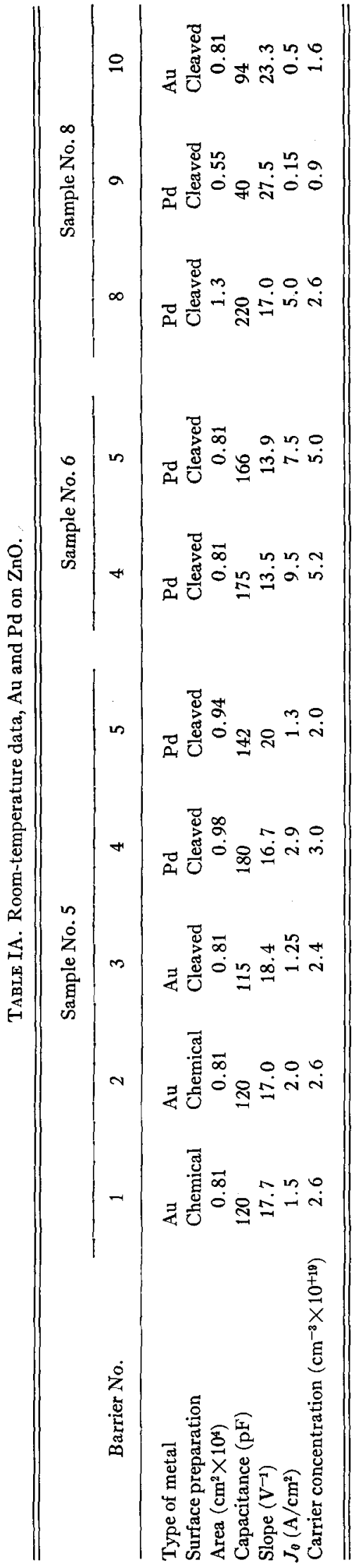


TABLE IIA. Liquid-nitrogen temperature data, Au and $\mathrm{Pd}$ on $\mathrm{ZnO}$.

\begin{tabular}{|c|c|c|c|c|c|c|c|c|}
\hline \multirow[b]{2}{*}{ Barrier No. } & \multicolumn{4}{|c|}{ Sample No. 5} & \multicolumn{2}{|c|}{ Sample No. 8} & \multicolumn{2}{|c|}{ Sample No. 6} \\
\hline & 2 & 3 & 4 & 5 & 1 & 2 & 4 & 7 \\
\hline Type of metal & $\mathrm{Au}$ & $\mathrm{Au}$ & Pd & $P d$ & $\mathrm{Pd}$ & $\mathrm{Pd}$ & Pd & $P d$ \\
\hline Surface preparation & Cleaved & Cleaved & Cleaved & Cleaved & Cleaved & Cleaved & Cleaved & Cleaved \\
\hline Area $\left(\mathrm{cm}^{2} \times 10^{4}\right)$ & 0.81 & 0.81 & 0.79 & 0.79 & 1.3 & 0.45 & 1.3 & 0.81 \\
\hline Capacitance $(\mathrm{pF})$ & 117 & 117 & 142 & 142 & 115 & 120 & 156 & 143 \\
\hline Slope $\left(V^{-1}\right)$ & 19.2 & 20.1 & 20.9 & 20.9 & 19.4 & 17.5 & 14.9 & 15.5 \\
\hline$J_{0}\left(\mathrm{~A} / \mathrm{cm}^{2}\right)$ & 1.6 & 1.25 & 1.0 & 1.0 & 1.9 & 4.5 & 12.0 & 9.0 \\
\hline $\begin{array}{l}\text { Carrier concentration } \\
\left(\mathrm{cm}^{-3} \times 10^{-19}\right)\end{array}$ & 2.5 & 2.5 & 2.0 & 2.0 & 2.4 & 2.6 & 3.4 & 3.4 \\
\hline
\end{tabular}

individual surface barriers for zero and small reverse bias. These values were used to determine the carrier concentration in the crystal at the localized region immediately under the surface barrier. Localized carrier concentrations between $7 \times 10^{18}$ and $5.7 \times 10^{19}$ per $\mathrm{cm}^{3}$ were obtained. Agreement between impurity carrier concentration derived from capacitance data and that determined from Hall and resistivity measurements was good.

The slope of the log current versus forward voltage curve was obtained as a function of the carrier concentration for room and liquid-nitrogen temperatures. This slope was then compared to the value for thermionic-field (at room temperature) and for pure field emission (at liquid-nitrogen temperature) current modes. The quantitative agreement is excellent at both room and liquid-nitrogen temperatures. The slopes were markedly different from that to be expected from pure thermionic currents. No evidence of tunneling via deep trapping levels was found.

For both room and liquid-nitrogen temperatures the magnitude of the current density is an order-of-magnitude less than that predicted by simple theory.

\section{APPENDIX A. DATA FOR GOLD AND PALLADIUM SURFACE BARRIERS ON ZINC OXIDE}

Tables IA and IIA below present pertinent data concerning gold and palladium surface barriers on zinc oxide at temperatures of $296^{\circ}$ and $77^{\circ} \mathrm{K}$, respectively. The values given are for specific barriers, but are typical of all the surface barriers measured.

Reverse currents were too large on this degenerate material to permit determination of impurity concentration from a $1 / C^{2}$ versus voltage plot. The authors used both $1 / C^{2}$ versus voltage and $C$ at zero bias methods on nondegenerate zinc oxide. ${ }^{2}$ Under those conditions results were in good agreement. Therefore, the effective donor concentration was calculated from the capacitance as measured at zero applied voltage and $1 \mathrm{MHz}$ as shown in the Eq. (1A)

$$
N_{d}=(2 / \epsilon q)\left(V_{b}\right)(C / A)^{2},
$$

where $\epsilon$ is the permittivity of zinc oxide at $1 \mathrm{MHz}$, $V_{b}$ is the effective barrier voltage, $C$ is the measured capacitance, and $A$ is the surface barrier area.

$A$ value of 8 has been assigned the dielectric constant after Collins and Kleinman. ${ }^{11}$ The barrier energies for gold and palladium are 0.66 and $0.60 \mathrm{eV}$ for chemically prepared surfaces. ${ }^{2}$ For cleaved surfaces values of 0.65 and $0.71 \mathrm{eV}$ have been assigned the surface barrier energies of palladium and gold (Ref. 1,2 ). The quantity $J_{0}$ is the extended intercept of the current density versus voltage characteristic for zero applied voltage.

The slope $S$ given is the slope of the logarithm of the current-versus-voltage curve, where after Eq. (3)

$$
J=J_{0} \exp S E
$$

where $J$ is the current density, $J_{0}$ is the current density extrapolated to zero bias, and $E$ is the applied voltage in energy units.

${ }^{1}$ C. A. Mead, Phys. Lett. 18, 218 (1965).

2 R. C. Neville and C. A. Mead, J. Appl. Phys. 41, 3795 (1970)

${ }^{3}$ F. A. Padovani and R. Stratton, Solid-State Electron. 9, 695 (1966).

${ }^{4}$ J. W. Conley and G. D. Mahan, Phy's. Rev. 161, 681 (1967).

${ }^{5}$ M. Millea, M. McColl, and C. A. Mead, Phys. Rev. 177, 1164 (1969).

${ }^{6}$ G. H. Parker and C. A. Mead, Appl. Phys. Lett. 14, 21 (1969).

${ }_{7}^{7}$ G. H. Parker, thesis, Calif. Institute of Technology, 1969.

${ }^{8}$ R. C. Deitz, J. J. Hopfield, and D. G. Thomas, J. Appl. Phys. 32, 2287 (1961).

${ }^{9}$ H. Rupprecht, J. Phys. Chem. Solids 6, 144 (1958).

${ }^{10} \mathrm{G}$. Bogner, J. Phys. Chem. Solids 19, 235 (1961).

${ }^{11}$ R. J. Collins and D. A. Kleinman, J. Phys. Chem. Solids 11, $190(1959)$.

${ }_{12}$ E. L. Murphy and R. H. Good, Jr., Phys. Rev. 102, 1464 (1956). 